DA040588 (T.P.). The latter two grants are part of the 4D Nucleome Initiative of the NIH Common Fund.

References: Crick, F.H.C. 1978. Chromosome structure and function. Future prospects. Eur. J. Biochem. 83: 1-3. Ma, H., Tu, L.-C., Naseri, A., Chung, Y.C., Grunwald, D., Zhang, S. and Pederson, T. 2018. Engineered CRISPR RNA scaffolds with enhanced stability for signal amplification in genome imaging. Nature Methods 15: 928-931. Mazia, D. 1987. The chromosome cycle and the centrosome cycle in the mitotic cycle. Intl. Rev. Cytol. 100: 49-92. Pederson, T. 1972. Chromatin structure and the cell cycle. Proc. Natl. Acad. Sci. USA 69: 2224-2228.

doi: http://dx.doi.org/10.7124/bc.0009BA

\section{Poly(ADP-ribose) polymerases in regulation of DNA repair}

\section{Olga Lavrik}

Institute of Chemical Biology and Fundamental Medicine, Novosibirsk State University, Novosibirsk, Russia

The phenomenon of nicotinamide adenine dinucleotide (NAD+)-dependent poly(ADPribosyl)ation catalyzed with PARP1 was discovered long time ago, but it is still unclear how this post-translational modification governs a multitude of cellular processes including DNA repair. When interacting with the damaged DNA, PARP1 catalyzes the synthesis of a long branched poly (ADP-ribose) polymer (PAR) by using NAD + as a substrate. PAR can be attached to the acceptor amino acid residues of nuclear proteins or to PARP1 itself. This process leads to reorganization of the functional protein complexes involved in DNA repair. The aims of the present study were to investigate the role of poly (ADP-ribosyl)ation in regulation of base excision repair (BER), to search new targets of PARylation catalyzed with PARP1 and PARP2 and to analyze the modulation of PARPs activity by RNA binding proteins. Methods: Biochemical approaches, fluorescence titration methods, atomic force microscopy (AFM), light-scattering technique. Results: PARP1 interacts with BER proteins as well as with DNA intermediates of BER containing breaks or apurinic/apyrimidinic sites [1]. PARP1 and PARP2 activities regulate BER. The PARP1 activity is modulated by disordered RNA binding proteins such as YB-1 and FUS. YB-1 stimulates PARP1 activity [2]. The PARP-1/PAR/DNA system was reconstituted in vitro and the role of FUS was analyzed at the single molecule level [3]. The dissociation of FUS from mRNA, its recruitment at DNA damage sites through its binding to PAR, and the assembly of damaged DNA-rich compartments were demonstrated. PARG, an enzyme family that hydrolyses PAR, is sufficient to dissociate damaged DNA-rich compartments and PARG hydrolysis initiates the nucleocytoplasmic shuttling of FUS in cells. We anticipate that FUS facilitates a rapid DNA repair through the transient compartmentalization of DNA damages by FUS interaction with PAR. Conclusion: The results obtained show the key role of PARP1 in regulation of BER and the modulation of PARP1 activity by RNA binding proteins YB-1 and FUS.

This work was supported by grant from RSF 19-14-00107 (study of PARPs activity modulation by RNA binding proteins) and by Russian State funded budget project of ICBFM SB RAS (AAAA-A17-117020210022-4).

References: 1. Moor NA, Lavrik OI. Protein-Protein Interactions in DNA Base Excision Repair. Biochemistry (Mosc) 2018, 83:411-422. 2. Alemasova EE, Naumenko KN, Kurgina TA, Anarbaev RO, Lavrik OI. The multifunctional protein YB-1 potentiates PARP1 activity and decreases the efficiency of PARP1 inhibitors. Oncotarget. 2018, 9:23349-23365. 3. Singatulina AS, Hamon L, 
Sukhanova MV, Desforges B, Joshi V, Bouhss A, Lavrik OI, Pastré D. PARP-1 activation directs FUS to DNA damage sites to form PARG-reversible compartments enriched in damaged DNA. Cell Rep. 2019, in press.

doi: http://dx.doi.org/10.7124/bc.0009BB

\section{The role of $\mathrm{NF}-\kappa \mathrm{B}$ transcription factor in cellular response to ionizing radiation}

Patryk Janus ${ }^{1}$, Katarzyna Szołtysek ${ }^{1}$, Gracjana Zając ${ }^{1}$, Wiesława Widlak ${ }^{1}$, Tomasz Stokowy ${ }^{2}$, Bartosz Wojtaś ${ }^{3}$, Piotr Widłak 1

${ }^{1}$ Maria Skłodowska-Curie Institute - Oncology Center, Gliwice, Poland; ${ }^{2}$ University of Bergen, Bergen, Norway; ${ }^{3}$ Nencki Institute of Experimental Biology, Warszawa, Poland

piotr.widlak@io.gliwice.pl

The NF- $\kappa \mathrm{B}$ transcription factor is involved in different aspects of the cellular response to stress, including atypical NF- $\kappa B$ pathway activated by damage induced by ionizing radiation. Moreover, NF- $\kappa \mathrm{B}$ could be involved in the regulation of genes activated by other stress-responsive factors. Here we aimed to perform the integrative genomics screening to compare subsets of NF- $\mathrm{KB}$-dependent genes induced by a pro-inflammatory stimulus and a high dose of ionizing radiation and also to identify new genes potentially co-regulated by NF- $\kappa$ B and p53 transcription factors in irradiated cells. Methods. The RelA-containing NF- $\mathrm{BB}$ dimers were activated by TNF $\alpha$ cytokine (classical proinflammatory pathway) and a single 4 or 10 Gy dose (atypical radiationinduced pathway) in human osteosarcoma cells. NF- $\kappa \mathrm{B}$-dependent and p53-dependent genes were identified using the gene expres- sion profiling (by RNA-Seq) in cells with downregulated RELA or TP53 combined with the global profiling of RelA and p53 binding sites (by ChIP-Seq). Candidate genes were subsequently validated by quantitative PCR. Results: There were $37 \mathrm{NF}-\kappa \mathrm{B}$-dependent protein-coding genes identified: in all cases RelA bound in their regulatory regions upon activation while downregulation of RELA suppressed their stimulus-induced upregulation, which apparently indicated the positive regulation mode (this set of genes included a few "novel" NF-kB-dependent species). The kinetics of the NF- $\kappa B$ activation was slower in cells exposed to radiation than in cytokinestimulated ones. However, subsets of NF- $\mathrm{KB}-$ dependent genes upregulated by both types of stimuli were essentially the same. Moreover, we identified a subset of radiation-modulated genes whose expression was affected by silencing of both TP53 and RELA, and a subset of radiation-upregulated genes where radiation stimulated binding of both p53 and RelA. For three genes an antagonistic effect of both transcription factors was observed: IL4I1 was activated by NF- $\kappa \mathrm{B}$ and inhibited by $\mathrm{p} 53$, while CDKN1A and SERPINE1 were activated by $\mathrm{p} 53$ and inhibited by $\mathrm{NF}-\kappa \mathrm{B}$. Moreover, RRAD was putatively co-activated by both factors. Conclusions: One could expect that similar cellular processes resulting from activation of the NF- $\mathrm{BB}$ pathway could be induced in cells responding to pro-inflammatory cytokines and in cells where so-called "sterile inflammation" response was initiated by radiation-induced damage. Moreover, certain stress-responsive genes induced by ionizing radiation could be co-regulated by NF$\kappa \mathrm{B}$ and $\mathrm{p} 53$. 